\title{
Publisher Correction: Seismic velocity reduction and accelerated recovery due to earthquakes on the Longmenshan fault
}

Shunping Pei $\mathbb{D}$, Fenglin Niu (D), Yehuda Ben-Zion, Quan Sun, Yanbing Liu, Xiaotian Xue, Jinrong Su and Zhigang Shao

Correction to: Nature Geoscience https://doi.org/10.1038/s41561-019-0347-1, published online 15 April 2019.

In the versions of equations (4), (5) and (6) originally published, the formatting of the vector terms was incorrect, dot product symbols were missing from the vector multiplications and line breaks were missing. These equations have been corrected to appear as below. Also, in the text following equations 4 and 5, these equations were mistakenly referred to as ' 74 ' and ' 75 ', respectively; this has now been corrected.

In addition, the authors wish to correct two further items: in the affiliations 'Earthquake Administration of Sichuan Province' should have been 'Sichuan Earthquake Administration'; in the Acknowledgements, the grant number '2016YFC0600303' for the National Key R\&D Program of China funding should have been '2017YFC1500303'. These errors have now been corrected.

$$
\begin{gathered}
\mathbf{A}\left[\begin{array}{c}
\delta \mathbf{s} \\
\mathbf{a}_{1} \\
\mathbf{b}_{1}
\end{array}\right]=\mathbf{A} \cdot \mathbf{x}_{1}=\delta \mathbf{t}_{1} \\
\mathbf{B}\left[\begin{array}{c}
\delta \mathbf{s} \\
\Delta \mathbf{s} \\
\mathbf{a}_{2} \\
\mathbf{b}_{2}
\end{array}\right]=\mathbf{B} \cdot \mathbf{x}_{2}=\delta \mathbf{t}_{2} \\
\begin{array}{c}
\left\|\mathbf{A} \cdot \mathbf{x}_{1}-\delta \mathbf{t}_{1}\right\|^{2}+\left\|\mathbf{B} \cdot \mathbf{x}_{2}-\delta \mathbf{t}_{2}\right\|^{2}+\lambda_{1}^{2}\|\mathbf{L} \cdot \delta \mathbf{s}\|^{2}+\lambda_{2}^{2}\|\Delta \mathbf{s}\|^{2} \\
\lambda_{2} \mathbf{L} \cdot \delta \mathbf{s}=0
\end{array}
\end{gathered}
$$

